\title{
versants
}

\section{William Ritter photograph(i)e}

\author{
Thomas BINGGELI \\ Université de Lausanne
}

\begin{abstract}
William Ritter multiplie les domaines d'activité et laisse derrière lui des archives dont il est difficile de faire façon. C'est notamment le cas pour la partie photographique de sa production : près de quatre mille clichés qu'il n'a jamais classés et qui reposent en vrac à La Chaux-de-Fonds, à Neuchâtel, et à Berne. Bien que méconnus, ces documents en disent beaucoup sur le monde dans lequel a évolué Ritter, et s'il ne prétend pas être un artiste photographe, il s'inscrit tout à fait bien dans un courant amateur très en vogue dès la fin du XIX ${ }^{e}$ siècle. Il documente ainsi son quotidien au gré de ses pérégrinations - en Suisse ou plus largement en Europe centrale - et laisse derrière des photographies dont les sujets sont variés, touchants, parfois très intimes et subversifs. Alors, comment les aborder, et quelle place leur accorder au sein de l'univers Ritter?
\end{abstract}

Keywords: William Ritter, photographie amateur, archives

Romancier, critique et amateur d'art, dessinateur, mélomane, voyageur curieux, photographe à ses heures : William Ritter est un touche-à-tout. De la Suisse romande aux quatre coins de l'Europe centrale, il accumule ainsi des documents en tous genres, publiés ou non, substantiels ou anecdotiques. Mais si son œuvre écrite a su retenir l'attention de ses contemporains et, dans une moindre mesure, de la postérité, le pan photographique de son activité demeure méconnu. Mentionnés çà et là dans la littérature critique, ces quelques milliers de clichés n'ont encore jamais fait l'objet d'une étude détaillée. Une brève plongée dans les fonds d'archives suffit pourtant à constater leur richesse. Paysages, natures mortes, portraits de familles, d'amis, d'amants, nus extravagants, détails d'architecture, inconnus, inconnues : la variété et le nombre de sujets représentés démontrent que cette pratique était chère à Ritter.

Face à cette matière abondante, les écueils méthodologiques se profilent bien vite. Les conditions de création et de conservation des fonds le rendent difficile d'accès et en entravent l'inventaire exhaustif. Cela nous force, pour l'heure, à nous contenter d'un nombre restreint de sources écrites sur la pratique photographique de Ritter, et plus généralement sur qu'il pouvait penser de cette forme relativement nouvelle de représentation du monde. Les quatre testaments successifs qu'il rédige entre I9I4 et I946 ne nous sont pas d'une grande aide : s'il y stipule ce qu'il conviendra de faire de ses biens après sa disparition, la photographie n'y apparaît pas une seule fois, comme si cette pratique ne constituait pas une trace significative de son passage, ou qu'elle appartenait uniquement au domaine privé voire intime. 
Si Ritter s'inscrit indéniablement dans une pratique amateur de la photographie très en vogue à son époque, quel sens donner à cette production? Photographier fait-il de lui un photographe ? Il s'agira de discuter plusieurs pistes de réflexion, aussi bien méthodologiques qu'interprétatives, photographies à l'appui. Bien entendu, il est probable qu'un carnet de note, un journal intime ou d'autres documents recelant un témoignage de première main refassent surface. Il s'agira, le cas échéant - surtout si le document présente des qualités théoriques plus abouties que ceux que nous possédons déjà -, de compléter la présente étude.

\section{Le legs photographique William Ritter : de quoi parle-t-on?}

Constitué entre I956 et la fin des années I970, les fonds William Ritter sont principalement conservés dans trois institutions suisses : la Bibliothèque de la ville de La Chaux-de-Fonds, la Bibliothèque publique et universitaire de Neuchâtel et la Bibliothèque nationale suisse à Berne ${ }^{\mathrm{I}}$. On doit cette tripartition à Josef Ritter-Tcherv (I904-I986), le dernier des trois amants de Ritter, que celui-ci adopte en I933. En tant que légataire universel, Tcherv se retrouve, dès I955, en possession de l'ensemble de la documentation de feu son «père » et endosse la responsabilité de sa transmission. C'est ainsi qu'il constitue différents lots et les remet par vagues successives aux institutions susmentionnées.

Seulement, Tcherv n'ayant pas privilégié d'unité thématique ou chronologique, on ne peut que faire dialoguer à tâtons les trois lieux dans l'espoir de compléter ce puzzle géant. Une telle division ne facilite pas les travaux de recherche de façon globale, et elle prétérite particulièrement le travail sur les photographies : estimées à plus de quatre mille, elles reposent en vrac dans chacune des trois villes et ne sont, pour la plupart, ni inventoriées, ni légendées, ni classées ${ }^{2}$. Mais comment expliquer un tel chaos ? Reconstituer l'historique des acquisitions n'est pas chose aisée car les registres présentent des lacunes. En revanche, il semble qu'au moins une partie des documents, dont les photographies, aient été vendus çà et là par Josef pour quelques milliers de francs. Une telle démarche n'est pas surprenante, compte tenu des difficultés financières du couple durant les dernières années de leur vie commune et de l'annulation, à la mort de Ritter, de la rente d'État salvatrice

I À ces trois bibliothèques s'ajoutent, semble-t-il, plusieurs institutions étrangères - la Bibliothèque nationale slovaque et le Musée de la littérature tchèque, par exemple - dont l'étendue des possessions n'est pas établie (inventaire rédigé par Xavier Galmiche pour le colloque L'Europe centrale en amateur: William Ritter, 2I-22 novembre 2008, Université Paris IV-Sorbonne).

2 Près de $80 \%$ des photographies sont conservées à Neuchâtel (un total d'environ 3200 clichés). La Chaux-de-Fonds et Berne se partagent à peu près équitablement les $20 \%$ restants. 
qu'il recevait depuis $1949^{3}$. Josef se voit donc dans l'obligation de subvenir seul à ses besoins, ce qui pourrait expliquer la vente incohérente de lots aux institutions intéressées et la division du legs telle que nous la connaissons.

Loin de jeter une lumière complète sur la question, la consultation des différents testaments de William Ritter est édifiante. Au nombre de quatre, ils sont rédigés entre I9I4 et I946 et discutent le sort de sa documentation après son décès. Dans le dernier, l'auteur dresse une liste de ses biens qu'il divise en six points. Alors que les points 2 à 5 traitent de legs faits à des membres de la famille ou qu'ils honorent certaines amitiés, il est intéressant de mettre en rapport les points I et 6. En effet, aussi précis soient-ils, les photographies n'y apparaissent pas une seule fois :

I Je lègue tous mes manuscrits, et surtout mon journal, commencé dès I882 et ininterrompu jusqu'ici, mes copie-lettres et correspondances (pleines d'autographes intéressants) à une bibliothèque publique, suisse et laïque, autant que possible la Nationale de Berne, ou les cantonales de Neuchâtel ou Lugano, indifféremment; mais le legs est indivisible (Ritter 1946).

6 Je voudrais que de mes dessins, aquarelles et pastels, il fut fait un ou divers choix que j'offre avec reconnaissance à tout musée, archives, ou bibliothèques publiques suisses qui en voudraient une portion. Beaucoups sont à conserver pour leur intérêt historique, puisque représentant des sites complètement évolués. - Les autres de mes travaux de peintre ou dessinateur sont à partager entre les enfants Montandon-Isenbart. Toutefois, je rends attentif à ce que tous les carnets de poches, notes de voyage, portraits d'amis, feuillets intimes, etc. rentrent dans la catégorie du legs sous chiffre un (Ritter 1946).

Comment quatre mille documents photographiques peuvent-ils avoir échappé à l'attention de Ritter ? Peut-on, sans imprudence, avancer que les photographies sont comprises dans cet équivoque et caetera et qu'elles étaient donc, aux yeux de Ritter, trop anecdotiques pour mériter leur place en toutes lettres dans ce papier ? Il paraît difficile de le démontrer. De plus, le testateur stipule clairement que cette partie du legs doit être traitée comme ses manuscrits, à savoir qu'elle est « indivisible ». Si les photographies sont

3 « [...] le conseil d'État du canton de Neuchâtel et la ville de La Chaux-de-Fonds, désireux de vous apporter une aide modeste, ont décidé, d'un commun accord, de vous faire parvenir un subside de 200 frs par mois au total, [...] à partir du I ${ }^{\mathrm{er}}$ avril I949. Le subside vous parviendra directement à chaque début de mois par l'administration postale. La décision ci-dessus est justifiée par le fait que vous avez apporté une contribution importante au développement des arts dans notre canton, grâce à vos travaux littéraires et à votre critique d'art, appréciés dans le monde entier. De son côté, la commune de La Chaux-de-Fonds a tenu à honorer par ce geste le souvenir de feu votre père, l'ingénieux Guillaume Ritter, à qui elle est redevable de son développement »(Donzé, Calame \& Charrière 1999 : 22). 
implicitement comprises dans cette sixième partie, force est de constater que cette consigne n'a pas été respectée.

Les deux hommes ont-ils résolu la question autrement ? Se sont-ils mis d'accord pour diviser le legs en cas de besoin, malgré la recommandation de Ritter de le garder uni ? Autant de questions qu'il est difficile de trancher à l'heure actuelle.

\section{Essor d'une pratique photographique amateur en Europe}

Quel que soit leur historique nébuleux, et même si elles n'avaient pas été, à l'époque, considérées comme une production d'importance majeure, ces photographies sont un outil de travail précieux, une occasion unique de plonger dans le - ou les - quotidien(s) de William Ritter. Mais que nous disent-elles de lui, exactement ? Ou plutôt, que peut-on leur faire dire?

La littérature spécialisée n'en parle que peu ${ }^{4}$, et il est tout aussi difficile de s'appuyer sur les propos théoriques de l'auteur car nous n'en possédons, pour l'heure, qu'un nombre restreint. Nous avons pourtant retrouvé deux textes intéressants : dans le premier, Ritter commente son approche photographique de la ville de Myjava (Ritter I9Io in Galmiche 20I9); dans le second, il fait un éloge passionné du photographe genevois Frédéric Boissonnas (I858-I946) (Ritter « Le voyage » [s.d.]). Il existe également plusieurs correspondances qu'il serait bon de classer et d'étudier plus en détail. La première est entretenue avec le même Boissonnas, mais semble essentiellement discuter la publication d'un des ouvrages de Ritter et quelques anecdotes personnelles. La seconde est entretenue avec Miloslav Rybák, un ancien étudiant de Ritter reconverti en aventurier, ethnologue et photographe amateur avec qui il voyage au début du $\mathrm{Xx}^{\mathrm{e}}$ siècle ${ }^{5}$. Enfin, il y la riche correspondance qu'entretiennent Ritter et Marcel Montandon, dans laquelle ils abordent parfois le sujet. Ce sont là, à l'heure actuelle, les pistes les plus prometteuses.

Mais peut-on pour autant avancer, sur la base de cette maigre matière, que la photographie n'était pas une préoccupation intellectuelle pour William Ritter ? La technique, à ses balbutiements durant la première moitié $\mathrm{du} \mathrm{XIX}^{\mathrm{e}}$ siècle, se perfectionne à partir des années I850. Dans un premier

\footnotetext{
4 Xavier Galmiche, par exemple, voit dans la pratique de Ritter une façon « d'attraper au vol une rencontre au hasard : un beau paysage, une scène pittoresque... ou un joli garçon ", mais ne s'étend pas sur le sujet (2008: I). Par ailleurs, le dictionnaire thématique de référence ne comporte pas d'article sur la photographie, à peine peut-on croiser le mot une fois ou deux (Donzé, Calame \& Charrière 1999).

5 Rybák est décrit comme un « étudiant » dans l'index de la correspondance conservée à la Bibliothèque nationale suisse de Berne. Les autres informations m'ont été aimablement fournies par le professeur Xavier Galmiche.
} 
temps, elle demeure une pratique artisanale nécessitant des connaissances techniques poussées et de l'argent, ce qui en fait l'apanage d'une élite aristocratique et bourgeoise. Elle se professionnalise, et les cabinets se multiplient pour devenir un commerce lucratif.

William vient donc au monde à l'heure où la photographie prend son essor en Europe. De plus, le cadre familial bourgeois dans lequel grandit le garçon favorise sa rencontre précoce avec ce nouveau média ${ }^{6}$. Si, comme l'avance Christian Joschke, les classes moins privilégiées doivent encore attendre des années avant de pouvoir y avoir accès, la photographie s'impose « au monde domestique bourgeois à la fin du XIX ${ }^{\mathrm{e}}$ siècle, comme atout essentiel de sa théâtralisation » (Joschke $2004: 54$ ). Cela semble être le cas pour la famille Ritter, ainsi qu'en témoignent les portraits d'enfant du petit William, réalisés entre I867 et I875, ainsi que les nombreux portraits de la famille élargie.

Reste que la majorité des photographies que nous possédons apparaissent à la fin des années I880, avant que leur nombre n'explose dès I900. Cette production coïncide avec l'invention et le développement des appareils photographiques instantanés, notamment ceux de la marque Kodak, déposée en I888. S'ils deviennent rapidement populaires aux États-Unis grâce à leur utilisation simplifiée, et à leur fameux slogan You press the button, we do the rest, ce n'est qu'en I897 que se diffuse un boîtier Kodak plus largement utilisé dans le monde occidental. Il s'agit du Kodak Folding Pocket, « premier appareil d'une longue série de foldings » et accompagné d'un format de papier de 2.25 par 3.25 pouces (soit 5,7 par 8,2 centimètres) qui restera une référence durant plusieurs décennies (Gustavson 2010 : I40). À en juger par le format de plusieurs de ses photographies, Ritter semble faire l'acquisition d'un appareil de ce type aux alentours de I900. Il peut ainsi facilement l'utiliser partout sans être freiné par la préparation de plaques de verres nécessitant des connaissances en chimie qu'il n'avait probablement pas.

Mais si William Ritter photographie, il ne semble bénéficier d'aucune formation théorique dans ce domaine, ni appartenir à un club dédié à cette pratique. Il ne semble pas non plus nourrir d'ambition artistique majeure ni, à ma connaissance, développer une forme de pratique professionnelle ou publique. Il ne vend pas ses clichés - de voyage, notamment - aux journaux et revues de l'époque, alors qu'il était courant de le faire. Cela corrobore les travaux de Christian Joschke, qui remarque qu'à partir des années I880,

on [voit] naître un intérêt artistique ou scientifique de la part d'usagers plus nombreux. Issus d'une bourgeoisie oisive et attirés par les horizons variés

6 Guillaume Ritter (I835-1912), le père de William, est un ingénieur érudit et curieux. Sa mère Joséphine est pianiste. Il ne serait pas surprenant que la photographie les aient intéressé(e)s dès ses débuts, et qu'il et elle en aient parlé à leur fils. 
de la pratique photographique, ils se regroupaient sous l'égide de sociétés nouvelles aux buts vagues, tels que "l'encouragement de la photographie amateur ». [...] Au cours d'une deuxième étape plus diffuse dans le temps (de I898 à I906 environ), les clubs de loisir photographique s'étendirent aux classes moyennes. Pharmaciens, médecins, avocats, mais aussi instituteurs, comptables, libraires ou autres commerçants se regroupaient en clubs qu'ils dotaient du matériel nécessaire : un agrandisseur, une chambre noire, parfois un projecteur de diapositives et une petite bibliothèque spécialisée (Joschke 2004:56).

Ritter souscrivait-il à cette vaste catégorie de photographes amateurs ? Comme il le dit dans l'introduction du texte qu'il consacre à Boissonnas, « il y a photographe et photographe comme il y a peintre et artiste » « Le voyage » [s.d.]). Bien qu'il ne fasse pas ici l'évaluation de son propre travail, il ne tarit pas d'éloges au sujet du photographe "de profession », qui a " mené les diverses techniques de son métier aussi haut qu'il était possible d'atteindre ». Boissonnas « joue du rayon lumineux non seulement avec la suprême maîtrise mecanique [sic], mais avec ce quelque chose d'indéfinissable et de réellement divin qui est le propre du génie ». Il sait «tire[r] de l'heure, du lieu, immédiatement - comme on gagne une bataille -, la seule image, le seul souvenir qui soient [sic] le définitif et le bon, parce que [mot illisible] le plus monumental et le plus poétique. Il n'est presque pas une en effet des grandes photographies rapportées par Boissonnas de ses excursions [...] qui ne soit avant tout une page de poète » («Le voyage » [s.d.]). À en juger par ces propos, Ritter fait une claire distinction entre le photographe artiste qu'est Boissonnas et le photographe qui ne serait que «peintre », qui ne ferait que pratiquer, sans atteindre cet état de création supérieur qu'il juge divin. Il se voit donc probablement appartenir à la large classe des amateurs, un groupe mouvant de curieux et curieuses qui voient en ce nouveau média une opportunité sans précédent d'expérimenter avec le réel et ses représentations, mais dont la production ne peut atteindre l'état de poésie divine qu'il identifie chez un homme comme Boissonnas.

Contrairement aux photographies amateurs publiques, la production de Ritter tombe dans une catégorie que Joschke et Sylvain Malfroy identifient comme privée, c'est-à-dire qui, « virtuellement porteu[se] d'une valeur documentaire inédite, reste cependant en retrait de la communication » (Traces 2004 : 9I-92). Ritter mêle ici photographies de voyage, portraits de famille, d'amis, et clichés plus intimes, privés, dont Malfroy dit qu'ils sont « [...] fort modestes quant à leur performance, mais riches d'un contenu humain introuvable ailleurs » (Traces $2004:$ 9I-92). 


\section{Panorama thématique}

\section{La maison et l'ailleurs : documenter le quotidien}

Il existe plusieurs portraits de groupe de la famille Ritter. Représentant souvent trois générations, ces clichés sont essentiellement réalisés à Monruz, en intérieur ou en extérieur. À partir de I903, ils incluent régulièrement Janko Cádra, le jeune amant rencontré en Slovaquie qui succédera à Marcel. Mais alors que la nature de leur relation pourrait choquer les valeurs passablement conservatrices des Ritter, on constate que Janko est bien intégré. Cette acceptation familiale n'est certainement pas sans lien avec l'assurance de William, un homme que Xavier Galmiche voit comme « l'exemple, après tout pas si fréquent à son époque, d'une homosexualité identifiée, assumée et vécue, toute la vie durant, parfois dans le bonheur, et assurément dans un certain épanouissement » (Galmiche 2008 : I) (fig. I \& fig. 2). Bien entendu, il serait naïf de croire qu'une relation homosexuelle ne pose de problème à personne. La présence officielle de Janko au sein de la famille - comme l'a été son départ de Slovaquie - était principalement justifiée par une forme de mentorat, un accompagnement intellectuel reléguant la nature sentimentale, érotique et sexuelle de leur relation au second plan, comme un «non-dit plutôt affectueux » (Galmiche $2008: 2$ ).

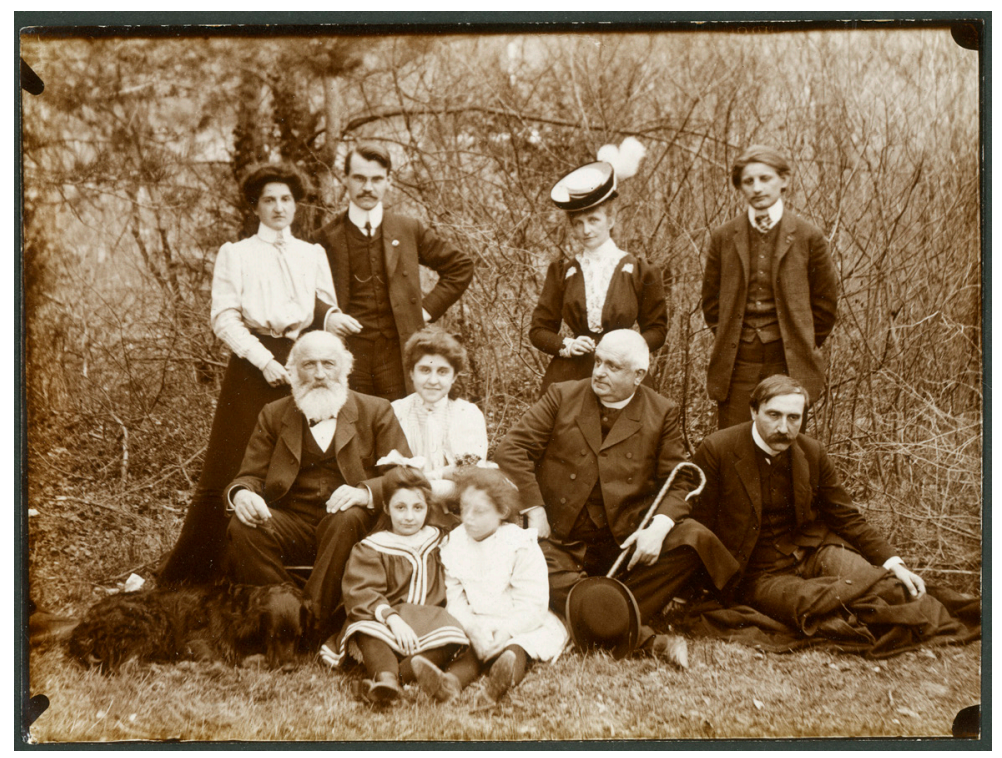

I. Photographe anonyme, Famille de William Ritter en extérieur avec Janko Cádra, I904I9I5, BPUN. 


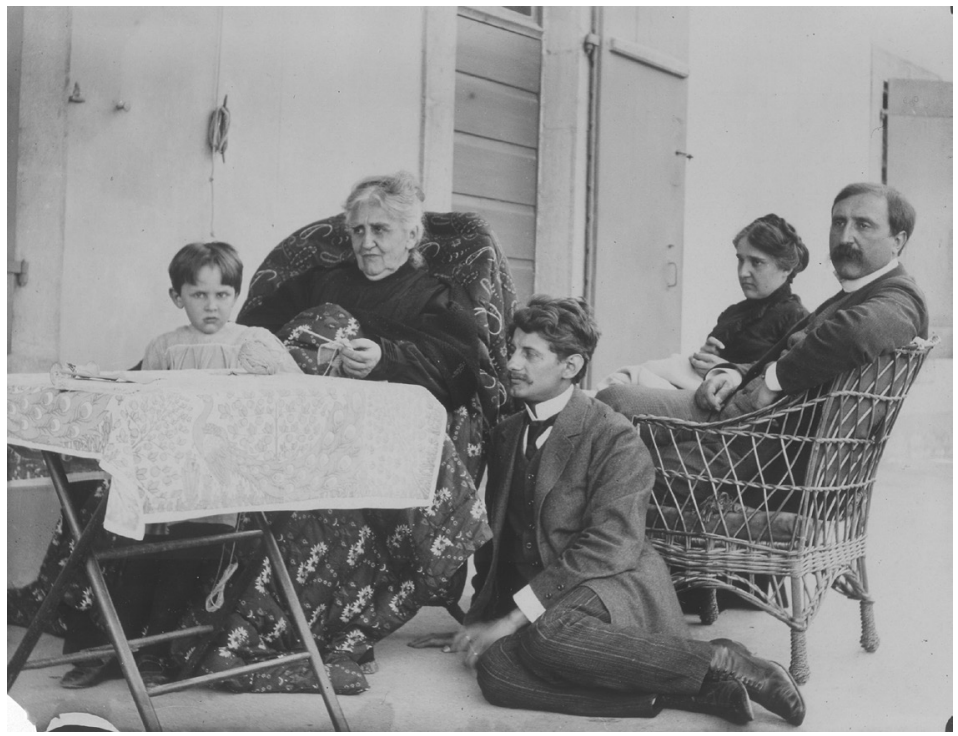

2. Photographe anonyme, William Ritter, Janko Cádra, Joséphine Ritter à Montruz, date inconnue, $\mathrm{CDF}$

Mais si Monruz est chère à Ritter, l'ailleurs est irrésistible. Ainsi, entre la fin des années I880 (date de ses premiers voyages en Europe centrale) (Donzé, Calame \& Charrière 1999 : 7) et le milieu des années I930 (qui marque le début de son indigence matérielle), il quadrille l'Europe au gré des rencontres, des envies, des amitiés qu'il noue ou délaisse. Tessin (fig. 3 \& fig. 4), Valais (fig. 5), Prague (fig. 6), Italie (fig. 7), Provence (fig. 8) : il documente ses pérégrinations avec la passion qu'on lui connaît, devant ou derrière l'objectif, c'est selon.

Cet amour pour la documentation visuelle vire à l'obsession en Slovaquie, pays dans lequel il retourne plusieurs fois au début $d u x^{e}$ siècle. C'est dans l'article intitulé " Architecture de Myjava ", peut-être rédigé en I9IO, qu'il justifie son besoin d'immortaliser un monde qu'il sait voué à disparaître (Ritter I9Io in Galmiche 20I9). Il débute son texte en évoquant le partage de ses clichés avec son ami Jeanneret, futur Le Corbusier : "Il ne sait rien, absolument du pays slovaque [sic], de la poésie de la campagne, de la majesté ou de la grâce millénaire de ses costumes ; rien de ses villages sans charmes, délicieux dans leur naïveté de si basse noblesse. » William « admire à Myjava des choses auxquelles personne ne prend garde » et veut transmettre cette richesse avant qu'elle ne soit plus : "Pauvre maison slovaque! Hâtons-nous d'en dessiner et d'en photographier les plus typiques exemplaires, puisqu'elle aussi est destinée à se modifier [...] » à cause, notamment, de « l'industrialisation et [des] machines » qui « auront tôt fait ici 


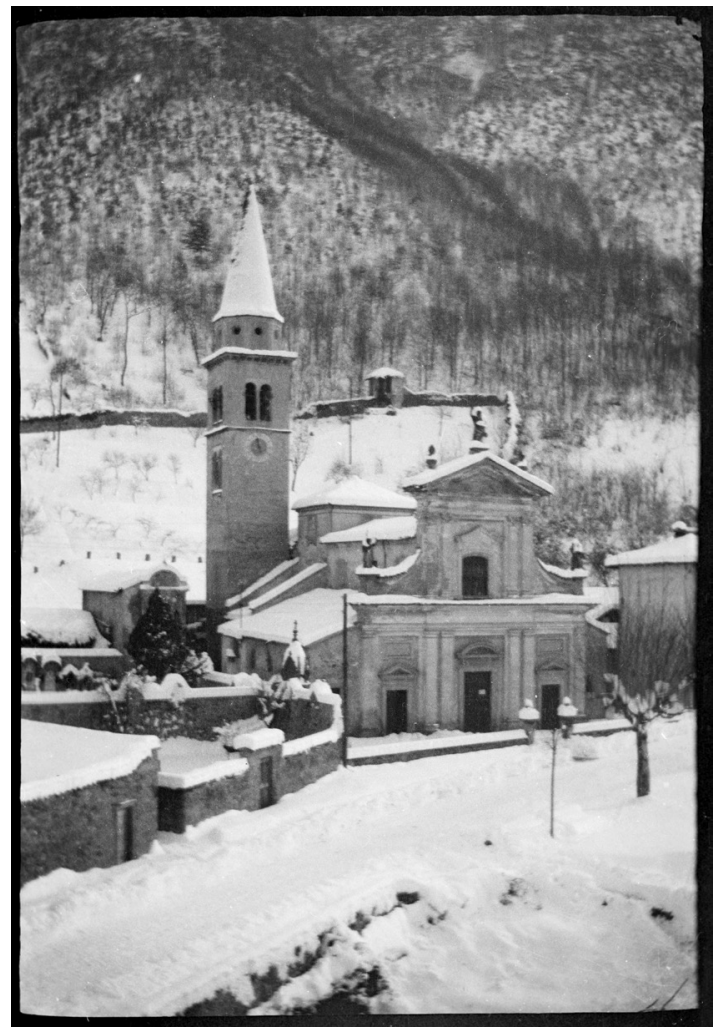

3. Photographe anonyme, Vue de l'église de Bissone, I926, BPUN

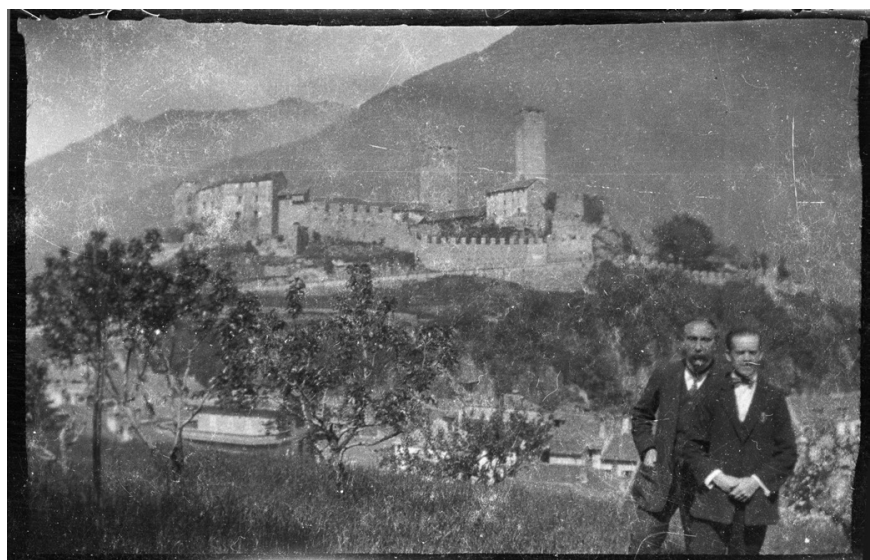

4. Photographe anonyme, William Ritter et Josef Tcherv devant le château de Bellinzone, 1925, 04, BPUN 


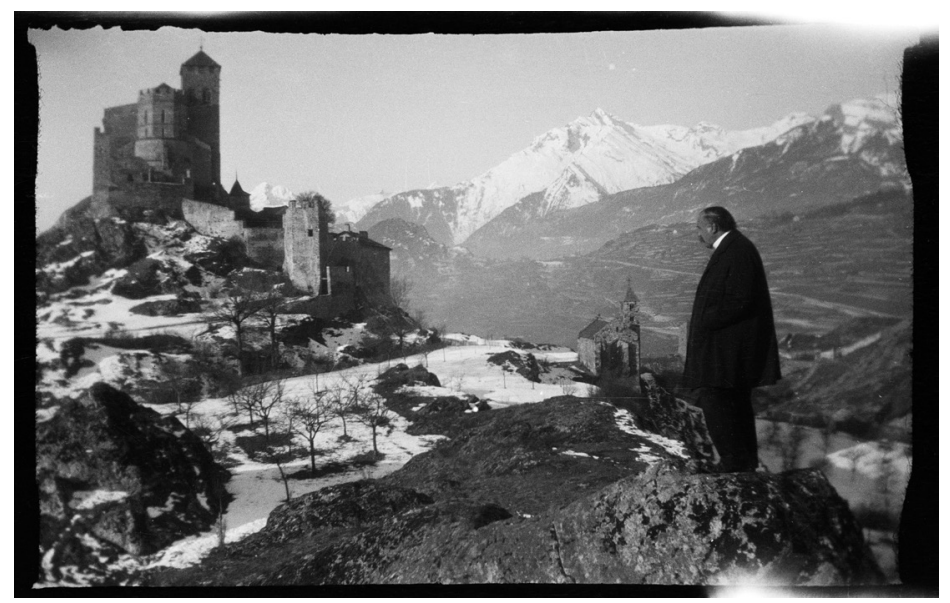

5. Photographe anonyme, William Ritter devant le château de Sion, 1928, Estimation de Josef Tcherv, BPUN

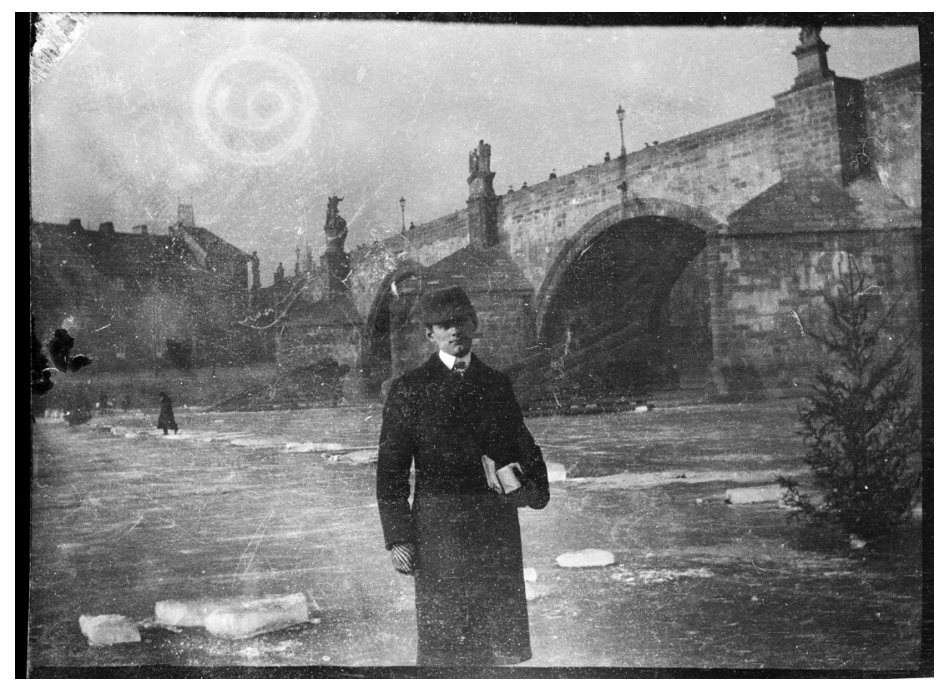

6. Photographe anonyme, Janko Cádra patinant sur [la Vltava] à Prague, 24.0I.I905, BPUN 


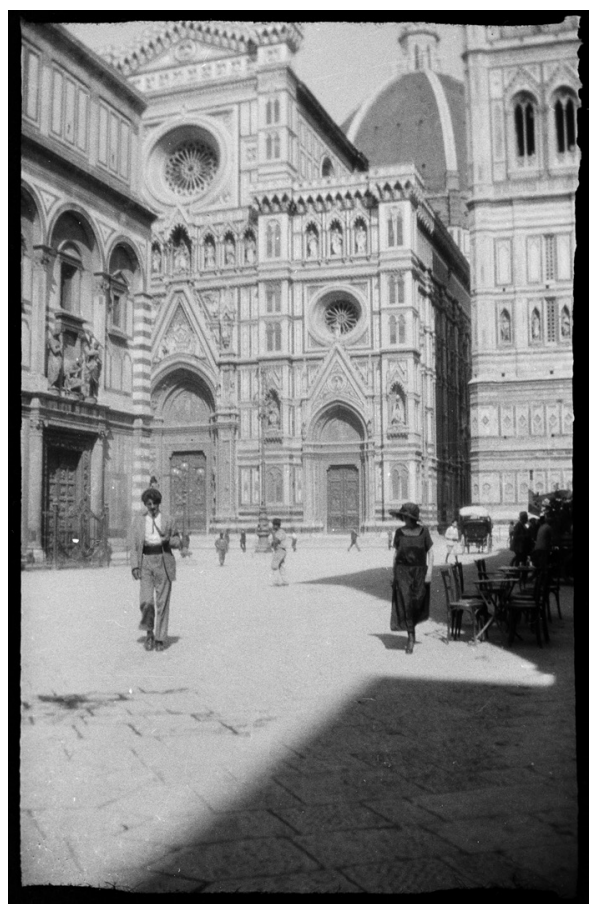

7. Photographe anonyme, Janko Cádra devant la cathédrale Santa Maria del Fiore à Florence, 1923 , BPUN

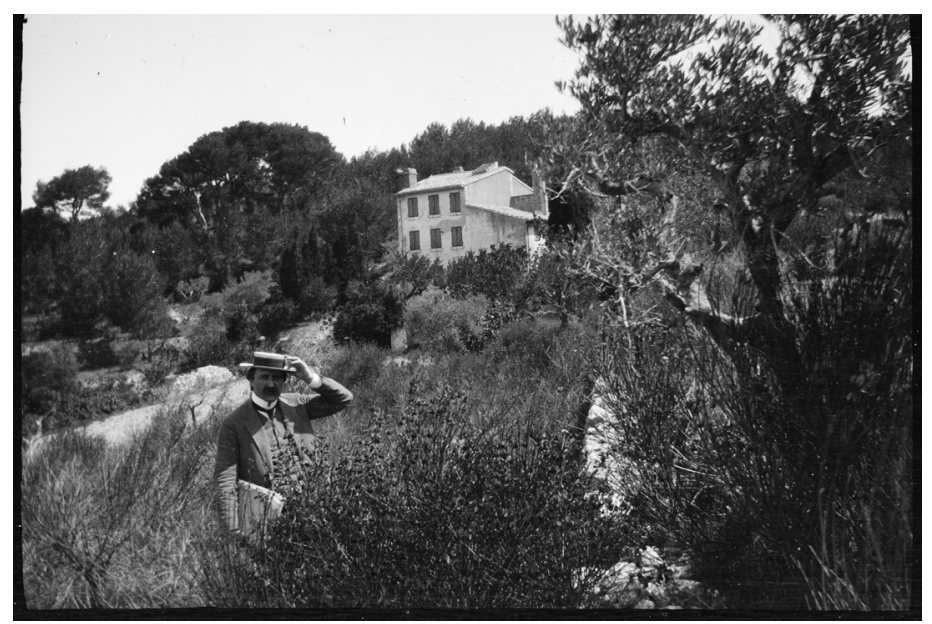

8. Photographe anonyme, William Ritter, avec son matériel de peinture, dans un champ devant une maison à Aix-en-Provence, 1907, BPUN 
comme en Hongrie de supprimer ces vieilles manières paysannes d'une si intense poésie » (Ritter I9IO in Galmiche 20I9). Il semble très affecté par le temps qui passe, par ce qu'il arrache de cher à tout un chacun. De ces bâtiments, magnifiques à ses yeux, il ne restera un jour plus rien, mais les immortaliser sur papier est un moyen efficace de combattre la fuite inéluctable du monde qu'il a connu :

Je les ai tous ou à peu près tous photographiés. Le moment venu où le vieux Myjava aura disparu, on en pourra retrouver le souvenir dans nos collections à Janko Cádra et à moi. [...] Le soin avec lequel je les ai dessinées et peintes et photographiées, la masse de documents que Janko Cádra et moi avons accumulés à leur sujet, est là pour prouver qu'il ne s'agit pas d'un amour platonique et qui se paie de mots. Tout cela tôt ou tard sera édité de façon à ce que nos après-venants puissent se rendre compte de ce que fut un jour le charme rustique slovaque de Myjava (Ritter I9Io in Galmiche 20I9).

Malheureusement, la majorité des photographies dont parle ici Ritter semblent perdues. Il ne reste, dans les cartons de la BPUN, que quelques exemples épars, comme cette photographie de lui en train de dessiner - une de ces maisons, peut-être? - assis au bord d'une route, en compagnie d'une fillette (fig. 9). Il y a cependant de beaux dessins, datés d'avril 1904 et réalisés à Myjava, représentant quelques exemples particuliers de ce que William définit comme une «pauvre maison slovaque » (fig. Io).

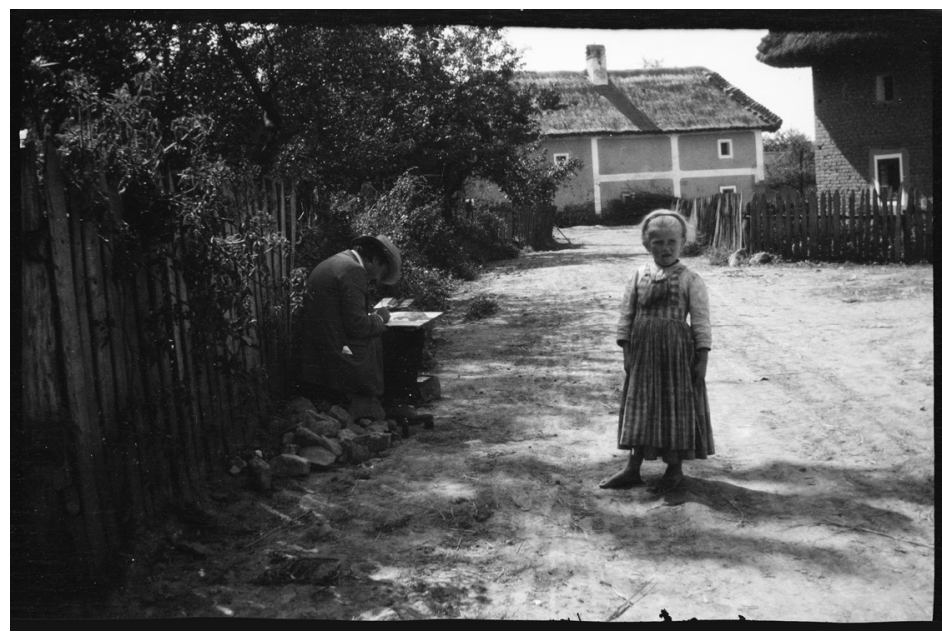

9. Photographe anonyme, William Ritter peignant à Myjava [rue Dofué Konec ?] (aujourd'hui Slovaquie) aux côtés d'une petite fille, or.09.1906, BPUN 


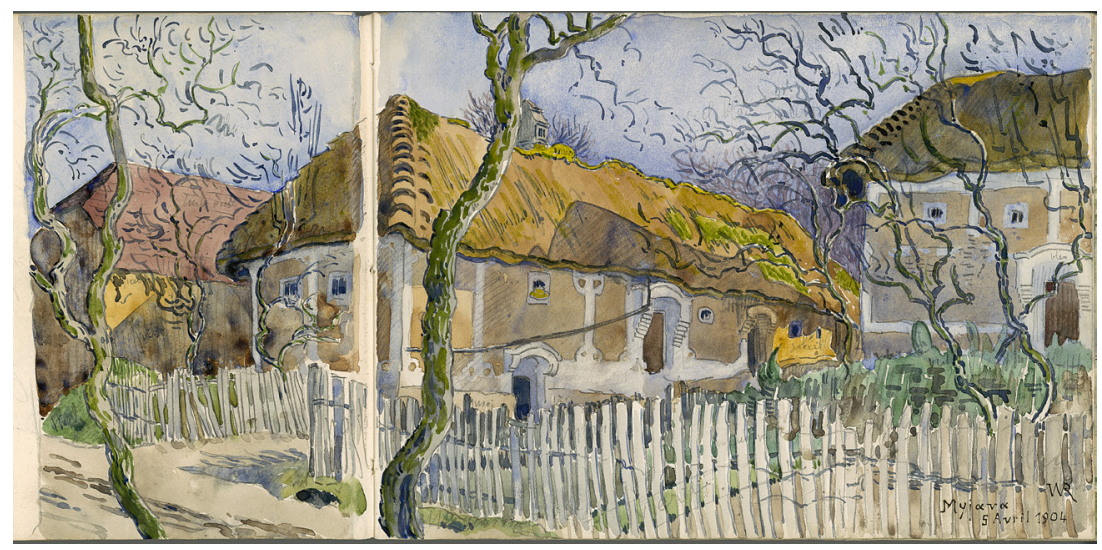

Io. William Ritter, «Myjava», croquis, 5 avril 1904, technique mixte, CDF

Il est clair qu'aux alentours de I9IO, Ritter avait l'intention de donner à voir « tôt ou tard » les photographies et le reste de la documentation ramenés de Slovaquie. Une telle publication n'a pourtant, semble-t-il, jamais vu le jour. Cela renforce le paradoxe créé par l'absence de mention des photographies dans les testaments et soulève une question majeure : que sont devenus tous ces documents? Il est difficile de croire qu'il ait pu les détruire ou s'en débarrasser, mais pourquoi renoncer à publier un album ou un récit de voyage?

\section{Les amants, les nus : immortaliser l'érotisme}

Si les photographies de Myjava se sont perdues, le fonds contient un nombre important de clichés plus intimes. Parmi eux, des portraits des trois grands amours de William : Marcel, Janko et Josef, qui se sont prêtés à de multiples jeux de pose et de mise en scène (fig. II, fig. I2 \& fig. I3).

Les clichés représentant Marcel sont moins nombreux, probablement à cause de la nature brève et plus ancienne de leur relation (entre I89I et I900) et parce que les appareils photographiques n'étaient, nous l'avons vu, pas encore aussi démocratisés (ou sophistiqués) que quelques années plus tard. Janko, en revanche, fait l'objet d'une véritable obsession de la part Ritter, particulièrement au début de leur relation. Infatigable, ce dernier réalise des centaines de croquis et photographies de son amant, habillé, dénudé, dormant, écrivant ou prenant des poses autrement plus suggestives. Josef, rencontré en 1923 , bénéficie d'une attention similaire, quoique les clichés 


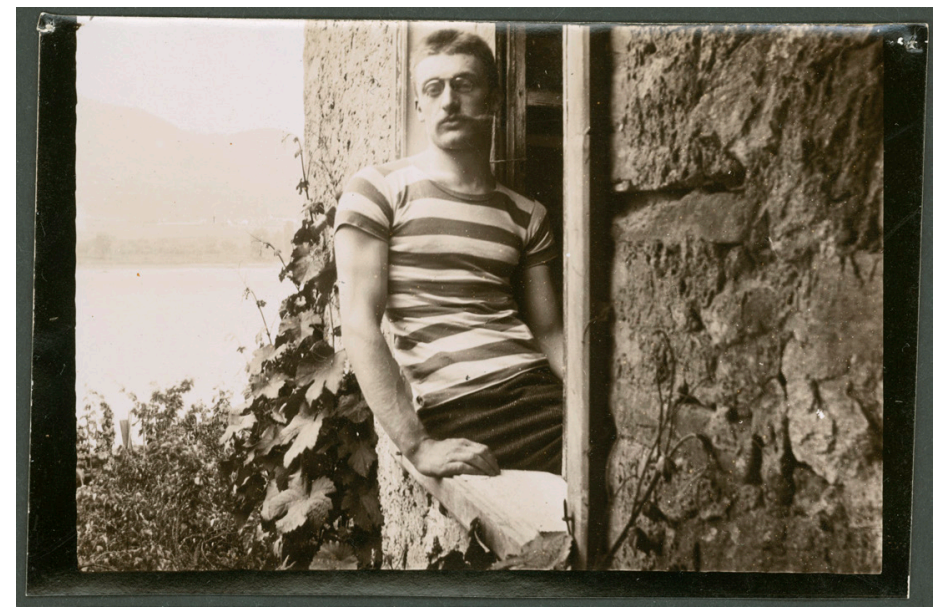

II. Photographe anonyme, Marcel Montandon en maillot rayé, 24.06.I899, BPUN

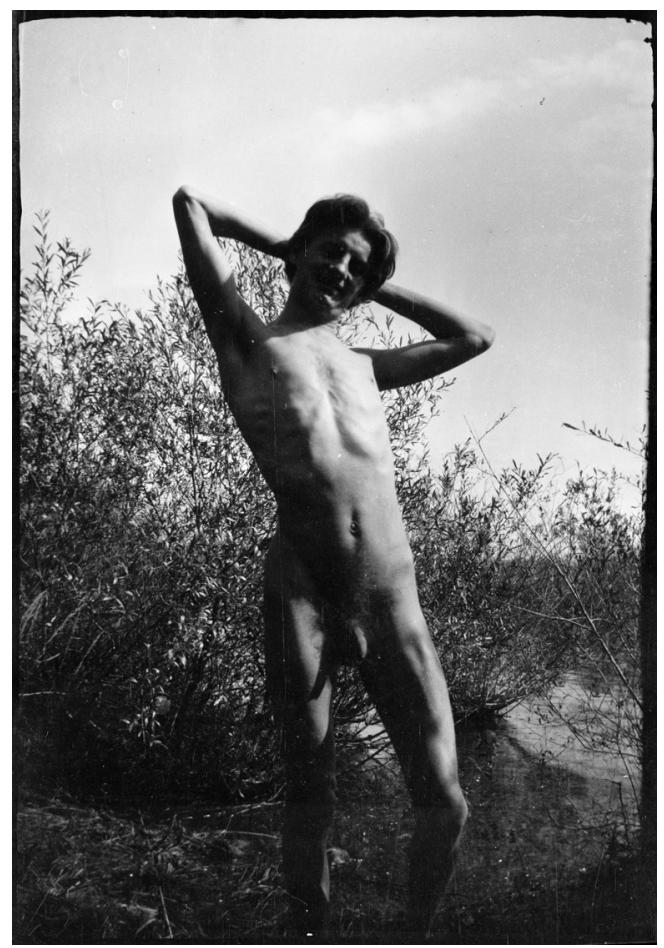

I2. Photographe anonyme, Janko Cádra, nu, s'apprêtant à se baigner, I923, o7, BPUN.tif 


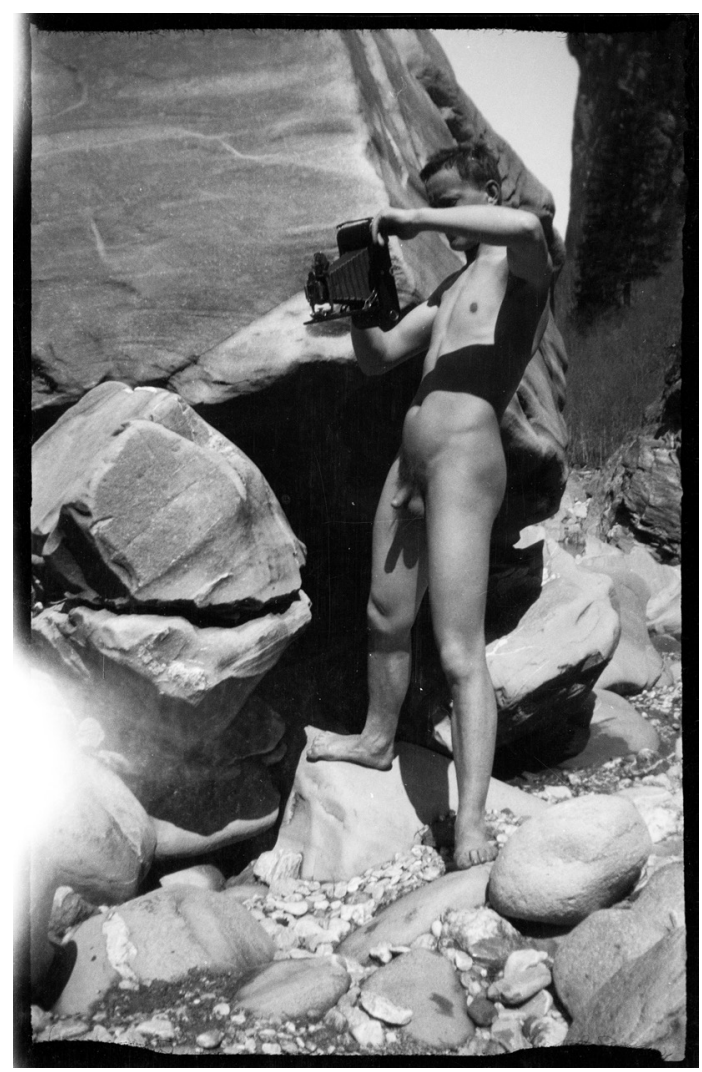

13. Photographe anonyme, Josef Tcherv, nu, prenant une photographie debout dans les rochers, I923-1927, BPUN.tif

le représentant soient un peu moins nombreux. C'est, d'une certaine façon, paradoxal, puisqu'il est celui qui passera le plus de temps avec William et survivra à tous les autres ${ }^{7}$. Par ailleurs, la photographie de Josef ci-dessus est particulièrement intéressante en ceci qu'elle est, à ma connaissance, la seule sur laquelle figure un appareil photographique. Il s'agit vraisemblablement aussi d'un modèle pliable de la marque Kodak: peut-être le même qu'au début du siècle, ou un modèle successif plus performant? Un tel cliché signifie aussi que deux appareils avaient été emportés par le groupe ce jour-là. À qui appartenait l'autre?

7 Il vivra seul avec William de 1927, date du décès de Janko, à 1955. Si Ritter se montre prolifique entre 1900 et 1935 , nous n'avons que peu de clichés des vingt dernières années de sa vie, et particulièrement de la période durant laquelle il vit à Melide. Cela pourrait s'expliquer par ses revenus très modestes, qui ne lui ont sans doute pas permis de faire des dépenses inconsidérées. 
Notons également que la rencontre de Josef ne semble pas altérer l'amour de William pour Janko. Très vite, le duo devient trio et aucun élément, à l'heure actuelle, ne pourrait laisser penser que les deux préférés ne s'appréciaient pas, ou qu'il entretenaient une quelconque forme de compétition (fig. I4). Les trois hommes passent donc quatre années heureuses à voyager, enseigner le français à Josef... et skier.

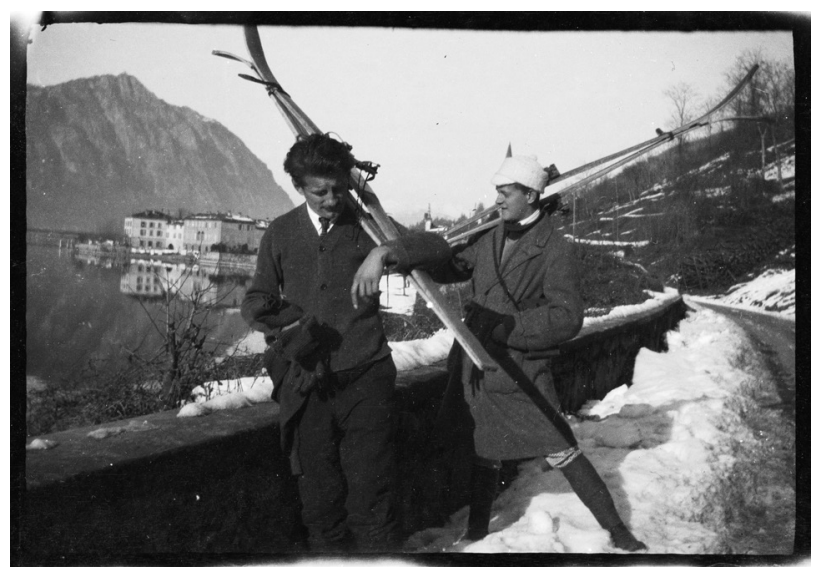

I4. Photographe anonyme, Josef Tcherv et Janko Cádra à ski, I923 - I927, BPUN

Mais en esthète et amoureux du corps masculin, William ne se contente pas de photographier ou de faire photographier ses amants. En témoignent les séries sur lesquelles figurent d'autres hommes passablement dénudés (fig. I5). Il est souvent difficile de les identifier, mais une grande partie des clichés a été réalisée durant les escapades lacustres de Ritter au Tessin en

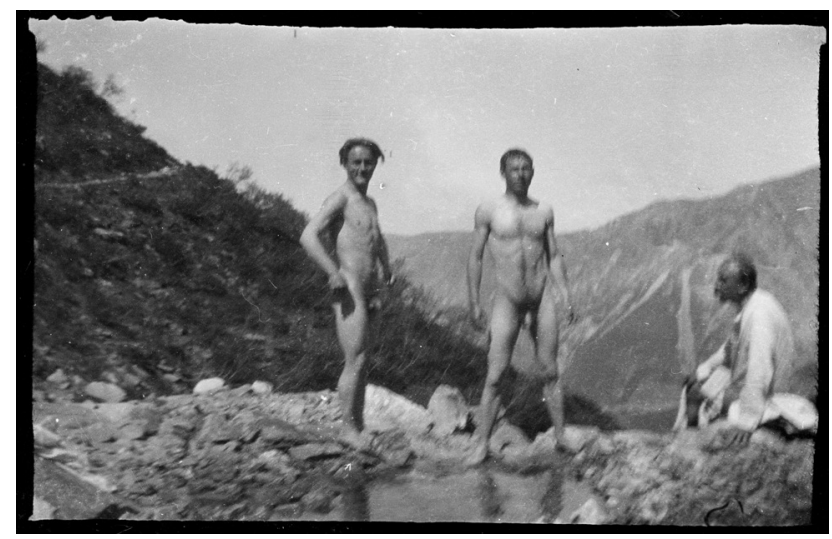

I5. Photographe anonyme, William Ritter assis en présence de Josef Tcherv (?), nu, et d'un autre homme, nu également, BPUN 
été, sans doute avec des amis d'accord de jouer le jeu. L'écrivain figure sur plusieurs d'entre elles, ce qui suggère qu'il a demandé à d'autres de le photographier en compagnie de ces garçons, ou simplement que les appareils passaient de main en main, en faisant de la photographie une activité légère et généreuse.

Malheureusement, Janko tombe gravement malade et décède en 1927. L'un des clichés montre William assis à côté de son buste, réalisé peu après sa mort (fig. I6). La sculpture semble contempler un Ritter à l'air mélancolique, presque abattu. L'amour profond que partageaient les deux hommes prend ici un aspect fantomatique, doublement immortalisé. Une telle mise en scène indique par ailleurs que l'image n'a pas été prise à l'insu de son modèle. Difficile, donc, de croire à sa spontanéité. Mais s'il peut paraître curieux de capturer un moment de deuil, il n'est pas impossible que ait souhaité étendre la documentation de son quotidien à de tels moments. Est-ce Josef derrière l'objectif?

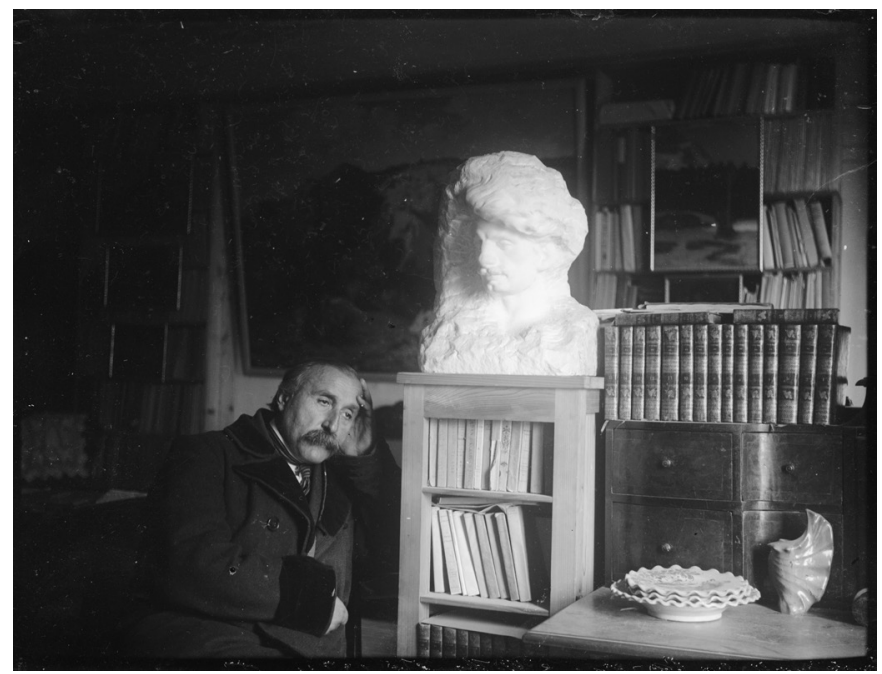

I6. Photographe anonyme, William Ritter devant le buste de Janko Cádra, en ou après I927, BPUN

Photographier, en somme, c'est se jouer de temps qui passe. C'est tordre le coup à cette force qui, peu à peu, emporte la pierre et la fermeté des chairs. C'est fixer sur papier le souvenir des êtres aimés et des belles choses, celles qui comptent, qui ont marqué l'imaginaire. C'est sans doute, comme l'écriture des journaux et la correspondance, le moyen de ne pas oublier. 


\section{Et le dessin?}

William Ritter photographie, écrit, mais ce n'est pas tout. Entre deux clichés, il passe aussi de nombreuses heures à dessiner. Prolifique, travailleur, inspiré - c'est sans doute son plus grand talent -, il croque tout le temps, partout, et ne se fatigue pas: le même objet d'étude peut devenir obsessionnel, comme le démontrent, par exemple, les croquis de Janko, les paysages aux alentours du lac de Lugano ou ceux de Slovaquie.

Deux photographies le montrent à l'œuvre muni de son matériel (fig. I7 \& fig. I8). Sur celle de gauche (fig. I7), on le voit en pleine nature, assis sur un petit siège pliable, une palette usagée traînant à son côté. Sur celle de droite (fig. I8), il est assis dans une barque, sous la silhouette de Josef. Ritter n'a donc pas peur d'emporter ses affaires même dans les situations les plus incongrues, ne serait-ce que pour jeter quelques traits de crayon dans un carnet en attendant d'ajouter les rehauts colorés dont il est friand (fig. Io). En effet, si la photographie de gauche nous montre qu'il emporte parfois son aquarelle, il est probable que certaines situations de plein air ne le lui permettent pas, le forçant à retravailler l'esquisse plus tard.

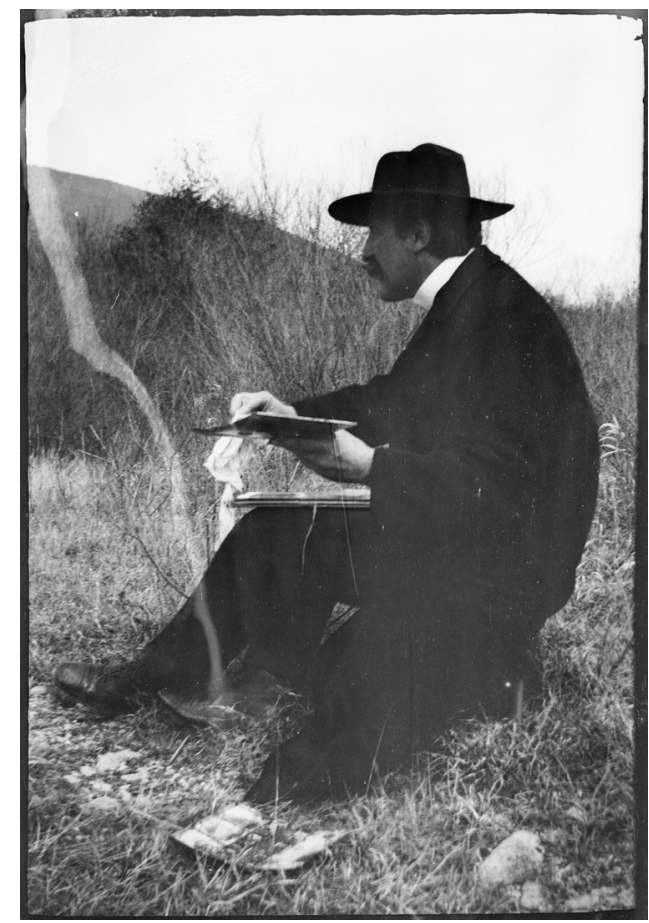

I7. Photographe anonyme, William Ritter dessinant, date inconnue, BPUN 


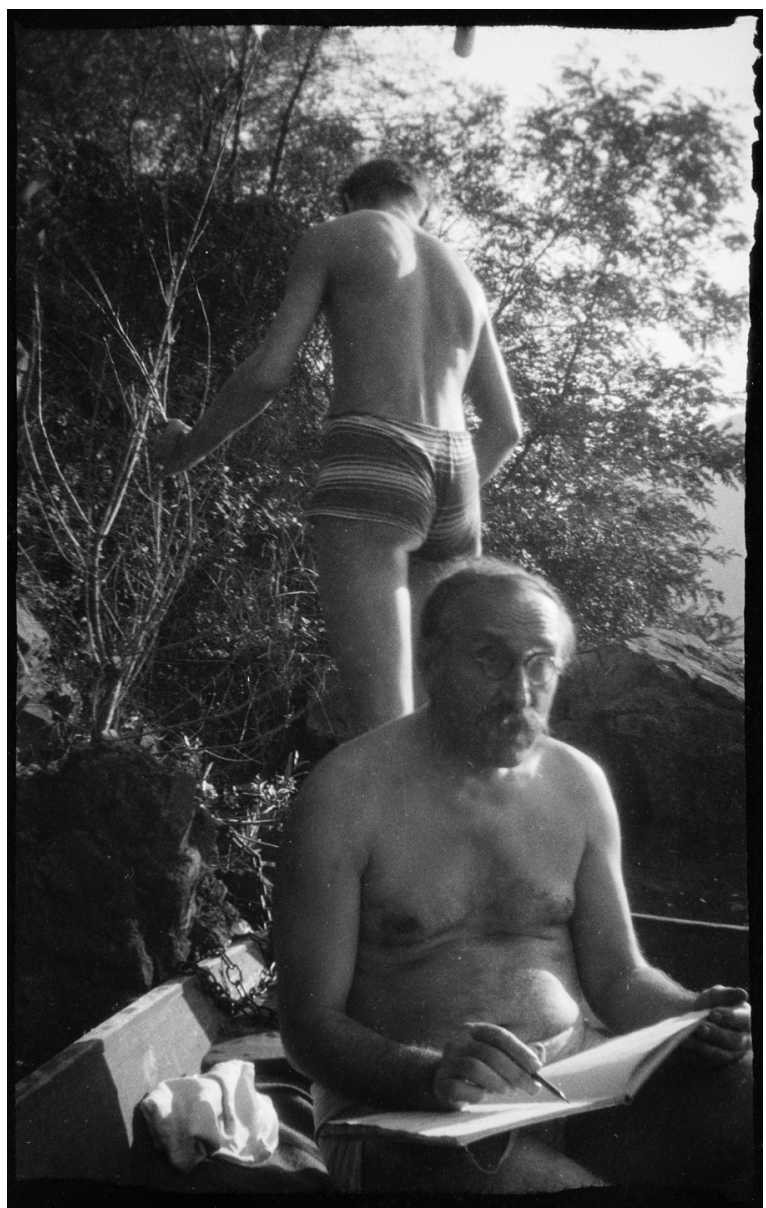

I8. Photographe anonyme, William Ritter peignant au bord de l'eau (lac de Lugano ?) et Josef Tcherv debout derrière lui, I924, BPUN

Par ailleurs, il est possible d'établir certaines correspondances troublantes entre ses productions photographique et graphique. On peut donc se demander si les photographies ne servent pas également de banque de données visuelles, si Ritter ne dessine pas d'après elles lorsque le temps presse ou que la situation ne se prête pas à une halte. Ces deux documents, par exemple, présentent des similitudes évidentes (fig. 19 \& fig. 20). Le bâtiment au fond est le même, et la date estimée de la photographie correspond à la datation du dessin. Le froid qui régnait l'a peut-être convaincu de prendre une photographie du lieu et de s'en inspirer pour réaliser ce paysage hivernal quelque temps plus tard. 


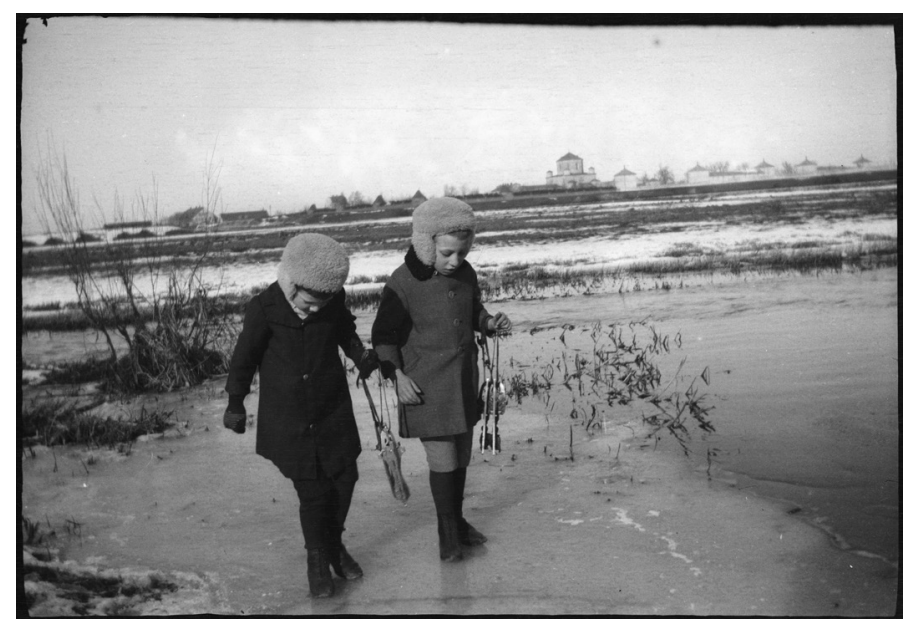

19. Photographe anonyme, Deux enfants sur une étendue gelée, à Munich, avec leurs patins, WILLIAM RITTER, I9OI-I9I4, BPUN

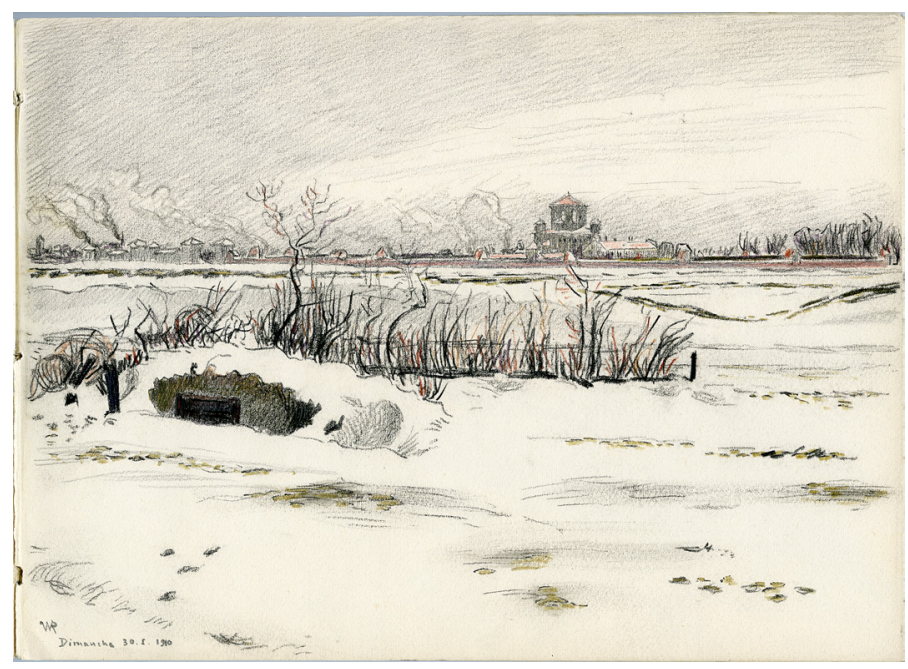

20. William Ritter, Étendue gelée à Munich, Dimanche 30.I.I9Io, croquis, CDF

Il existe également de nombreux dessins de ces barques tessinoises reconnaissables à leur armature arrondie. La ressemblance entre ce croquis non daté (fig. 2I) et cette photographie de Josef Tcherv (fig. 22) est particulièrement frappante : il pourrait bien s'agir de la même expédition lacustre. Puisqu'emporter son matériel de dessin sur l'eau ne semble pas l'effrayer, il se peut que Ritter ait réalisé ce croquis au milieu du lac, pendant que 


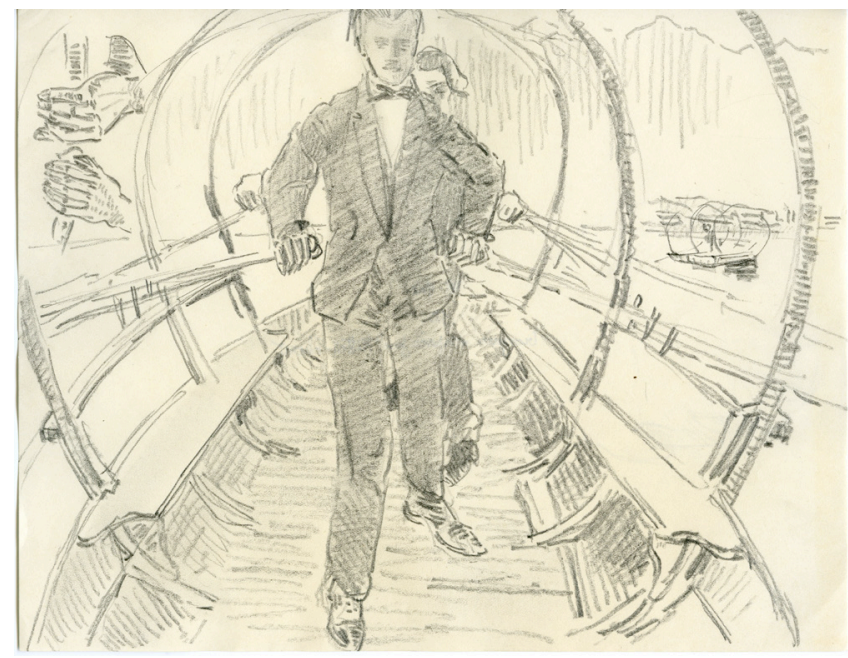

2I. William Ritter, Josef Tcherv ramant, croquis, sans date, CDF

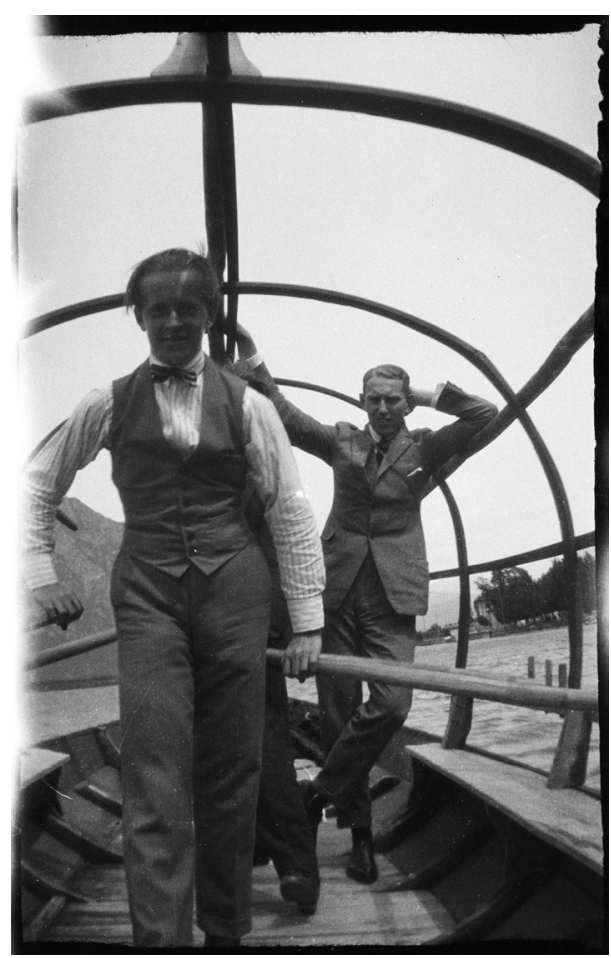

22. Photographe anonyme, Josef Tcherv ramant, sans date, BPUN 2 
Josef pagayait. Cependant, l'habillement et les positions de Tcherv et de l'homme derrière lui sont légèrement différents suivant le médium : Ritter aurait pu photographier la scène puis la reproduire au crayon pour s'entraîner. Il en va de même pour ces deux représentations de Janko (fig. 23 \& fig. 24). Malgré la différence de vêtement, la ressemblance est indéniable : le chapeau, la barque, la banquette sur laquelle il est assis, la position de son corps, l'arrière-plan, tout correspond. Seulement, la photographie est datée du 28 août 1907, un mois après le dessin, qui lui est daté du 29 juillet.

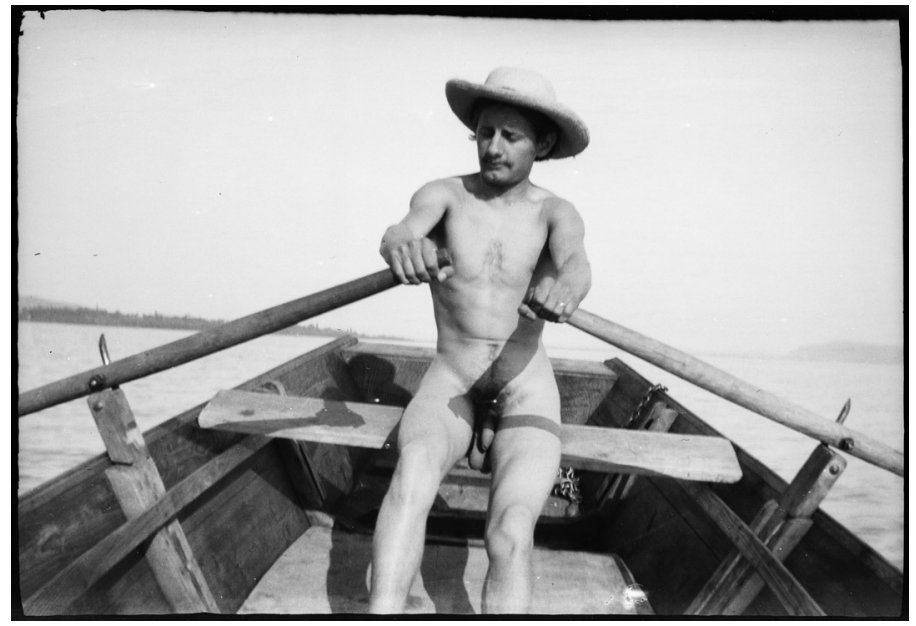

23. Photographe anonyme, Janko Cádra, nu, ramant, 28 août I907, BPUN

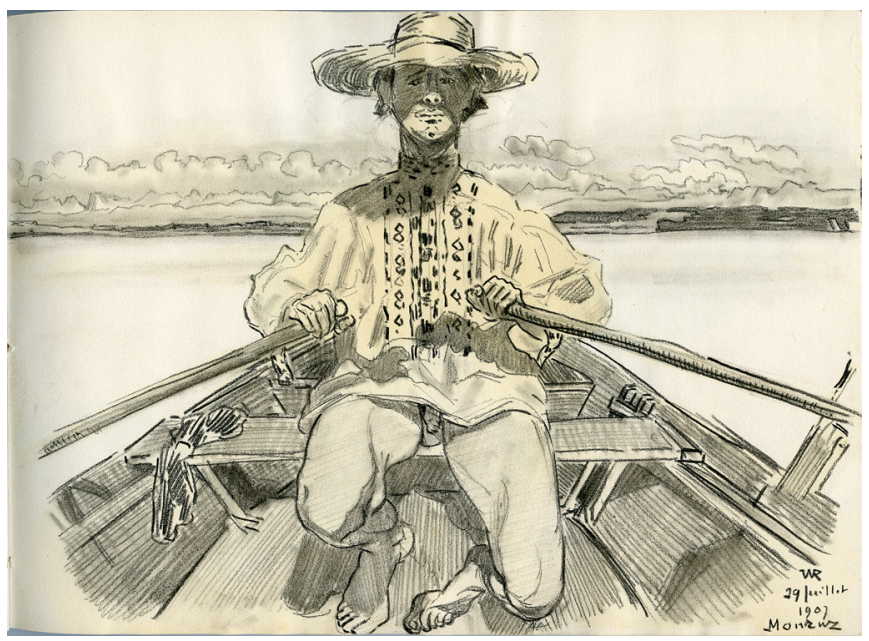

24. William Ritter, Janko Cádra dénudé, ramant, 29 juillet I907, CDF 2 
Si l'on se fie à ces datations, le dessin n'a pas pu être inspiré de la photographie, mais l'erreur n'est pas impossible. Le soldat assis sur l'armature d'une de ces barques, reconnaissable au chapeau qu'il a seul gardé de son uniforme, soulève le même type de questionnement (fig. 25 \& fig. 26).

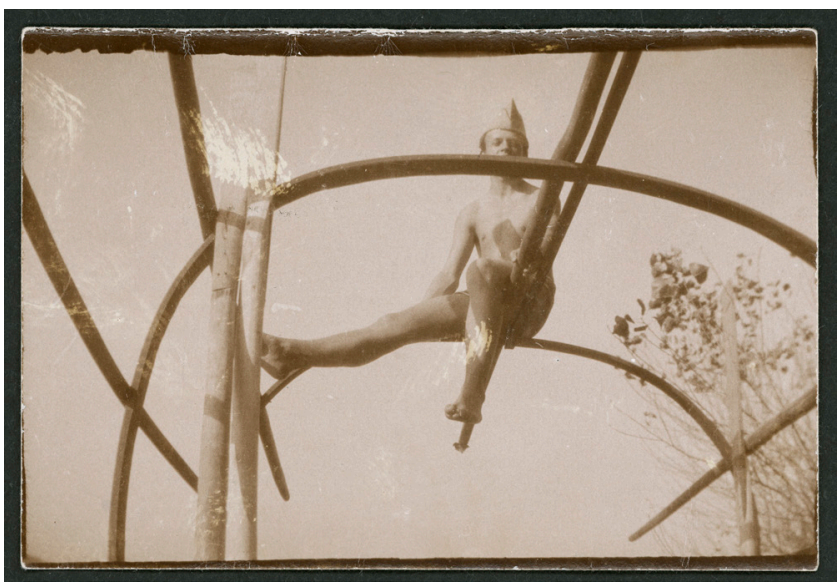

25. Photographe anonyme, Homme nu sur l'armature d'une barque tessinoise, sans date, BPUN

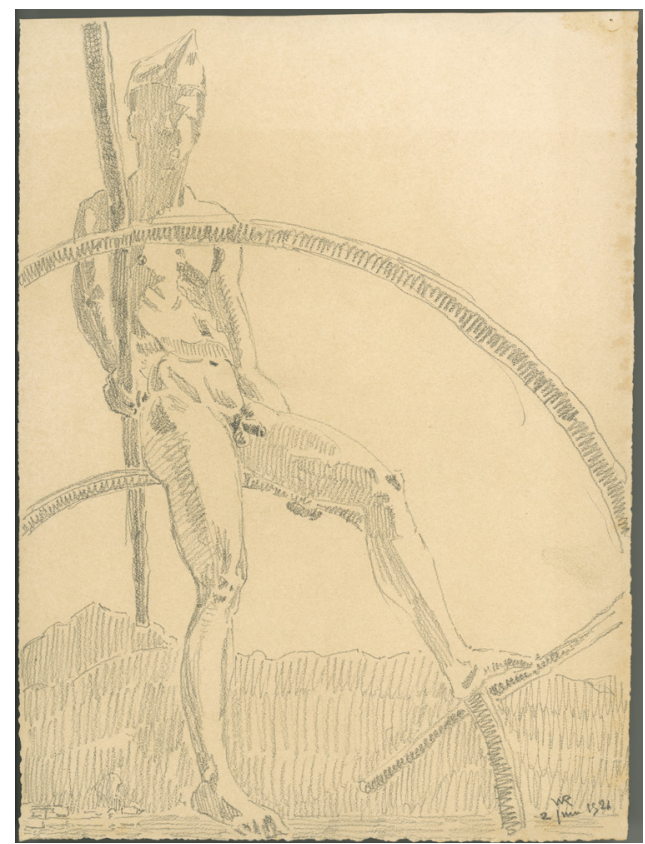

26. William Ritter, Homme nu sur l'armature d'une barque tessinoise, croquis, 2 juin 1926 
Mais s'il semblait, jusque-là, difficile d'affirmer la nature utilitaire de ces correspondances, la découverte fortuite du dessin suivant laisse penser que la piste est bonne (fig. 27). La légende indique qu'il a été réalisé en 1938 , "d'après une photographie ». Celle-ci pourrait donc avoir été prise quelque temps plus tôt à ces fins graphiques, tout comme Ritter pourrait être retombé dessus des années plus tard et se sentir inspiré. Impossible, en revanche, de la retrouver.

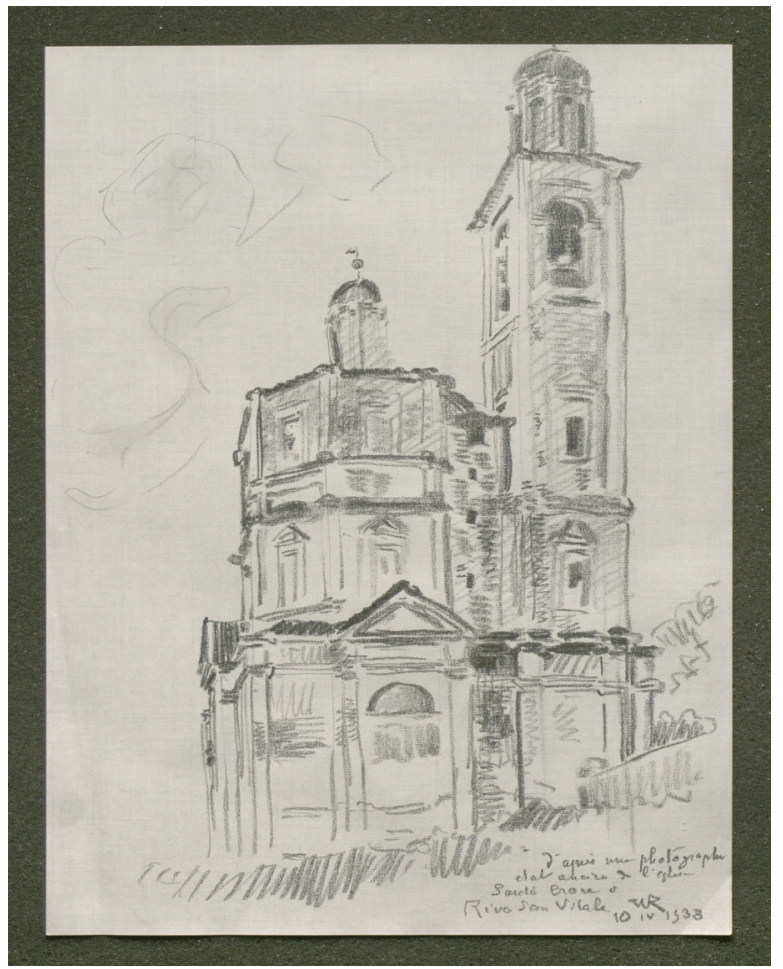

27. William Ritter, D’après une photographie / état ancien de l'église Santa Croce à Rivo San Vitale, croquis, Io IV I938, BPUN

La mémoire est inconstante, certains détails plus marquants que d'autres. Pourquoi reconnaître un bâtiment minuscule au fond d'une étendue gelée à Munich et probablement manquer une cathédrale connue ou un portrait ? D'autres liens pourraient être faits, mais ceux-ci suffisent à avancer que le dessin et la photographie sont deux pratiques complémentaires pour William Ritter, deux moyens parallèles d'assouvir un même besoin d'archivage du monde qui l'entoure. À en juger par ses testaments, il accordait simplement plus d'importance à son activité de dessinateur, peut-être plus noble à ses yeux, ou d'une plus grande valeur esthétique. 


\section{Créer l'œuvre photographique?}

Boîte après boîte, dossier après dossier, faire défiler photographies, dessins, carnets de notes, trier, parcourir lettres et index, aller, venir. Fouiller, parfois au hasard, parfois moins, dans l'espoir de trouver le document, la pièce manquante d'un puzzle que l'on a imaginé, d'abord timidement, mais que l'on se surprend à vouloir compléter, rendre cohérent. Créer des liens, faire dialoguer des choses qui ne sont a priori pas liées, bâtir des ponts, hésiter. C'est bien le problème de ces fonds : en l'état, des centaines d'heures de recherche n'en viendraient pas à bout sans laisser planer un doute important sur l'exhaustivité de la démarche.

Afin de permettre un travail plus systématique, il conviendrait de compléter celui entrepris par la BPUN qui, en 2016, a mis en ligne une sélection pertinente de 300 photographies dont chacune des cotes reflète une unité thématique ${ }^{8}$. Ainsi, les clichés seraient accessibles en fonction des douze catégories établies dans ce système ${ }^{9}$. Cela permettrait d'y voir plus clair, mais il resterait à déterminer quel statut leur accorder au sein de l'univers Ritter. Cependant, établir la valeur d'un fonds d'archives et de ses composantes n'est pas chose aisée. Jacques Derrida a, en son temps, beaucoup réfléchi à la question de l'archivage et à ce qu'une telle démarche implique :

C'est un geste de pouvoir. [...] dès qu'il y a une institution, il y a des personnes qui sont appointées et qui ont compétence reconnue pour contrôler l'archive, c'est-à-dire pour choisir ce qu'on garde et ce qu'on ne garde pas, ce à quoi on donne accès, à qui on donne accès, quand et comment, etc. Il n'y a pas d'archives sans cette organisation quasi étatique, en tout cas légitime et politique, du matériau ainsi informé, c'est-à-dire auquel on donne forme justement par l'interprétation et la classification, la hiérarchisation, la sélection. Il n'y a pas d'archives sans destruction, on choisit, on ne peut pas tout garder. Là où on garderait tout, il n'y aurait pas d'archives. L'archive commence par la sélection, et cette sélection est une violence. Il n'y a pas d'archives sans violence (Derrida 2014 : 60).

Ici, la violence consisterait à choisir à la place de William Ritter. En sortant les photographies du purgatoire où elles reposent depuis des décennies, on leur imposerait l'autorité qu'il ne leur a pas conférée de son vivant. On déciderait qu'elles ont ou n'ont pas de valeur esthétique, que certaines

8 Cet aperçu est disponible sur la plateforme Memoriav : http://memoriav.ch/projects/ fonds-william-ritter/ ?lang=fr.

9 Ces catégories sont, dans l'ordre : Portraits de William Ritter, Janko Cadra et Josef Tcherv; À Monruz ou en famille ; Portraits de proches et de connaissances ; Lieux de résidence ; Séjours en Suisse ; Séjours en Europe de l'Est ; Séjours en Italie ; Séjours en France ; Séjour en Allemagne ; Baignades et scènes de nudité ; Ethnographie ; Collection de photographies de tiers. 
d'entre elles pourraient faire l'objet d'un travail approfondi alors que d'autres ne seraient que des doublons, des développements ratés, flous, peu intéressants formellement, dont le contenu ne serait pas interprétable. On se lancerait dans un premier exercice de classification avant d'entamer, davantage que dans ce texte, un processus de sélection et d'interprétation. Il s'agirait de créer un corpus de référence qui pourrait toucher un plus large public, d'élaborer un catalogue et, pourquoi pas, de monter une exposition. Ce faisant, tout un pan de la production de William Ritter serait donné à voir, et la décision serait prise : on ne dirait plus que William Ritter photographie, car entre-temps, il serait devenu William Ritter, photographe.

Cependant, n'y a-t-il pas quelques considérations morales à avoir lorsque l'on décide de créer une œuvre posthume sur la base de documents intimes ? Derrida, à nouveau, demande : «Où doit s'ar rêter la collecte, la mise en ordre et la mise à la disposition publique de l'archive ? » Si l'on en croit Ritter, il a réalisé les clichés de Myjava à des fins documentaires et a souhaité, pendant un temps du moins, les rendre publics. La question des clichés intimes est, en revanche, plus complexe. Leur auteur ne les a pas détruits : ne l'aurait-il pas fait précautionneusement si l'idée de leur transmission lui était insupportable ? Étant donné le soin qu'il a apporté à l'élaboration de ses archives, il paraît peu probable qu'il ait « oublié » l'existence de ces photographies. Peut-être étaient-elles réellement anecdotiques à ses yeux - à moins qu'il ne s'agisse d'une énième provocation.

Peut-on, en définitive, étudier William Ritter sans embrasser la complexité kaléidoscopique de sa production ? Si la critique fait principalement de lui un auteur - un homme qui écrit, au sens large -, les fonds d'archives offrent une perspective plus vaste. Son côté touche-à-tout ferait presque de lui un artiste-archiviste. Difficile, d'ailleurs, de segmenter les différents pans de sa production : une fois que l'on s'y plonge, les correspondances et les liens entre tout ce qu'il entreprend sautent aux yeux. Graphomane compulsif, il donne son avis sur tout, tout le temps, au risque d'agacer et de déplaire. Mais ce qui l'emporte, c'est son enthousiasme pour la vie, pour ce qu'elle offre d'agréable, de bouleversant. Tous les moyens sont bons pour capturer ces instants-là, ne pas les laisser filer. Son attrait pour la photographie n'est donc pas étonnant : complémentaire à son travail d'écriture, elle fait partie intégrante de sa quête documentaire et est, en ce sens, fondamentale à la compréhension de ce personnage singulier. William a aimé documenter ses aventures grâce à cet art de l'instantané, comme on le fait aujourd'hui. Plus qu'aimer cela, photographier semble avoir été un besoin profond. S'attendait-il à ce que ses images soient, plus de soixante ans après sa mort, publiées dans une revue suisse lui étant consacrée? 


\section{Bibliographie}

Ritter, William, "Architecture de Myjava ", I9Io [?], in Galmiche, Xavier, William Ritter voyage en Slovaquie (I903-1914) - Album d'un pays rêvé, Paris, Eur'ORBEM éditions, 2019 (à paraître).

—. «Le voyage en Grèce d'un photographe-artiste. Frédéric Boissonnas », Ms. inédit, s.d.

—. [M'étant recueilli en présence de Dieu...], « Testament », Ms inédit, 1946.

\section{Littérature critique}

Berchtold, Alfred, «William Ritter », in La Suisse Romande au cap du xx siècle, Lausanne, Payot, 1963, pp. 828-834.

Derrida, Jacques, Trace et archive, image et art, Bry-sur-Marne, Institut National de l'Audiovisuel, Collection Collège iconique, 2014.

Donzé, Fernand, Calame, Caroline \& Charrière, Edmond, William Ritter au temps d'une autre Europe : dictionnaire à l'usage des curieux de la vie et de l'oeuvre d'un Neuchâtelois hors du commun, Neuchâtel, Nouvelle Revue neuchâteloise (numéro monographique), 6I, 1999.

Frizot, Michel, Nouvelle Histoire de la photographie, Paris : Bordas, 1994.

Galmiche, Xavier, «William et les garçons (d'Europe centrale) : "Aventures de volupté sous d'autres cieux" ", in Actes du colloque "L'Europe centrale en amateur. William Ritter (I867-I955) ", 2I-22 novembre 2008, Paris, Centre interdisciplinaire de Recherches centre-européennes, www. circe.paris-sorbonne.fr/spip.php?articlei94.

-.William Ritter voyage en Slovaquie (I903-I9I4) - Images d'un pays rêvé, Paris, Eur'ORBEM éditions, 2019 (à paraître).

Gustavson, Todd, 150 ans d'appareils photo. Histoire de la photographie du daguerréotype au numérique, New York, Sterling Publishing, 2010.

Joschke, Christian, « Aux origines des usages sociaux de la photographie. La photographie amateur en Allemagne entre I890 et I9IO ", Paris, Actes de la recherche en sciences sociales, 2004/4, $\mathrm{n}^{\circ} \mathrm{I54}, \mathrm{pp}$. 53-65.

Traces : Ioo ans de patrimoine photographique en Suisse, Neuchâtel, Institut suisse pour la conservation de la photographie, 2004. 